\title{
ASSESSMENT OF ABOVEGROUND BIOMASS STOCK IN THE PACHAIMALAI FOREST OF EASTERN GHATS IN INDIA
}

\author{
ARUL PRAGASAN, L. \\ Department of Environmental Sciences, \\ Bharathiar University, Coimbatore - 641046, India \\ (phone: +91-422-2428392) \\ e-mail: arulpragasan@yahoo.co.in \\ (Received 31 $1^{\text {st }}$ May 2013; accepted 14 ${ }^{\text {th }}$ Aug 2014)
}

\begin{abstract}
Forest biomass plays a vital role in the global carbon cycle. The aim of the present study was to determine aboveground biomass of trees in the Pachaimalai forest of the Eastern Ghats in India. A sum of 5388 trees representing 131 species were recorded in twenty four transects ( 0.5 ha each) sampled from Pachaimalai. The total aboveground biomass stock for the 12 ha sampled was 608.2 tonnes. The average biomass value per transect was $25.3 \pm 5.6 \mathrm{t} / 0.5 \mathrm{ha}$, and it ranged from 4.2 to $103.5 \mathrm{t} / 0.5$ ha. The average species richness, density and basal area per transect were $22 \pm 1.7$ (species/ $0.5 \mathrm{ha}$ ), $225 \pm 9.5$ (trees $/ 0.5 \mathrm{ha}$ ) and $6.3 \pm 1.0\left(\mathrm{~m}^{2} / 0.5 \mathrm{ha}\right)$ respectively. Among the 131 species, Nothopegia heyneana (Anacardiaceae) contributed the maximum (19.1\%) to the total forest biomass followed by Canthium dicoccum var. dicoccum, Albizia amara, Gyrocarpus asiaticus and Schleichera oleosa. Linear regression analysis showed that forest biomass increased with the increase in basal area $\left(R^{2}=0.979\right)$ and species richness $\left(R^{2}\right.$ $=0.702)$, but not with density $\left(R^{2}=0.320\right)$. The aboveground biomass of Pachaimalai forest falls within the range reported for other tropical forests of the world. The present study provides valuable data for the estimation of carbon stock of Pachaimalai forest, which would facilitate carbon stock modelling at national as well as at global level.
\end{abstract}

Keywords: biomass; Eastern Ghats; India; Pachaimalai forest; regression analysis

\section{Introduction}

Global climate is changing day by day as a result of natural variability and anthropogenic activities. Changes in the atmosphere, water cycle, solar inputs and land surface are evident (Negi et al., 2003; Alamgir and Al-Amin, 2008). Among the few global issues that have received more attention from scientists, resource managers, policy makers and public is climate change (Tiwari and Singh, 1987). Increase in carbon emission has become a major concern, which was well addressed in the Kyoto Protocol because it is the main causal factor in global warming (Alamgir and Al-Amim, 2008).

Tropical forests represent 30-40\% of the terrestrial net primary productivity (Mitra et al., 2011). The rapid conversion of tropical forests was a major source of greenhouse gases such as carbon dioxide, methane, and nitrous oxide (Nascimento et al., 2002). The trees act as a major $\mathrm{CO}_{2}$ sink which captures carbon from the atmosphere and store the same in the form of fixed biomass during the growth process (Chavan and Rasal, 2010).

Carbon stock includes aboveground biomass measurements as an essential aspect (Ketterings et al., 2001). Forest biomass can be determined either by direct methods or by indirect methods. Direct methods or destructive methods involve felling of trees to determine biomass (Salazar et al., 2010). Destructive techniques for biomass estimation are time consuming and expensive due to the large dimensions and the amounts of biomass that have to be processed and also against conservation (Verwijst and Telenius, 1999). Indirect means of estimation of forest biomass are based on allometric equations,

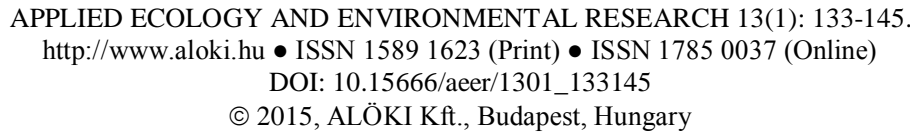


using tree inventory data (Brown et al., 1989). Allometric relationship is the most preferred method since it yields a non-destructive and indirect measurement of biomass components and it also consumes less time and less expensive (Nath et al., 2009).

Allometric equation is a crucial step in the estimation of tree biomass, yet it is seldom directly tested (Crow, 1978; Brown et al., 1989; Houghton et al., 2001; Chave et al., 2005). Since, a hectare of tropical forest may be home to as many as 300 different tree species and one cannot use species-specific regression models, as in the temperate zone (Shepashenko et al., 1998; Brown and Schroeder, 1999).

With regard to the estimation of tree biomass, allometric equations using the diameter of the tree are a strong predictor of biomass for practical purposes (Salazar et al., 2010), owing to the ease and precision with which it can be obtained and because it relates the total volume of biomass to functional processes such as transport and the age of the tree (Satto and Madgwick, 1982). In the other hand, allometric equations using tree height along with tree diameter is also used, but it does not improve the results, besides it represents an additional hindrance in data compilation (Salazar et al., 2010). Further, it is often difficult to measure the height in dense forests (Segura, 2005). Hence, tree diameter alone was used as the independent variable for the estimation of forest biomass in most of the earlier studies.

Forest biomass data are available for different regions of the world (Murphy and Lugo 1986; Brown et al., 1989; Laurance et al., 1997; Clark and Clark, 2000; Mani and Parthasarathy, 2007; Salazar et al., 2010), but the biomass stock of the Pachaimalai forest of the Eastern Ghats in India was not estimated. Hence, the present study was undertaken to determine the biomass stock in Pachaimalai forest.

\section{Materials and methods}

\section{Study area}

The present study was undertaken in the Pachaimalai forest of the Eastern Ghats in

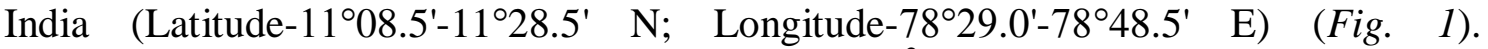
Pachaimalai forest covered an area of about $586 \mathrm{~km}^{2}$, altitudes ranged from $200 \mathrm{~m}$ to $1021 \mathrm{~m}$ above mean sea level. The study area composed of masses of Charnockite associated with gneisses and varied metamorphic rocks, and soil was red, loamy and rich in aluminum and iron oxides (Pragasan and Parthasarathy, 2010). Climate data such as the mean annual temperature and rainfall during the 1988-2007 (20 years) for Salem, the nearest station to Pachaimalai forest were $28.3^{\circ} \mathrm{C}$ and $1058 \mathrm{~mm}$, respectively. The bulk of the rainfall was received from August to October, and the mean annual rainy days were 61 days. Tribal settlements were common in Pachaimalai forest. The hilly terrain and the surrounding plains of Pachaimalai forest were densely populated. Increasing human population, encroachment of forest land, expanding agriculture, poor harvesting methods and over-exploitation of wild species besides the invasion of the weed species such as Lantana camara constitute major threats to the study area.

\section{Methods}

The Pachaimalai forest was divided into twenty four grids of size $6.25 \mathrm{~km} \times 6.25 \mathrm{~km}$. Within each grid, a transect of 0.5 ha $(5 \mathrm{~m} \times 1000 \mathrm{~m})$ area was laid, and all the trees with $\geq 30 \mathrm{~cm}$ girth at breast height $(1.37 \mathrm{~m}$ from the ground level) were inventoried (Pragasan and Parthasarathy, 2010). The inventory data was used for determination of 
biomass stock in Pachaimalai forest. The aboveground biomass of trees were estimated through allometric equation using tree diameter following Brown et al. (1989), Y = $34.4703-8.0671 \mathrm{D}+0.6589 \mathrm{D}^{2}$, where ' $\mathrm{Y}$ ' represents biomass per tree in $\mathrm{kg}$, and ' $\mathrm{D}$ ' is diameter in $\mathrm{cm}$. Regression analysis was performed to find the relationships of forest biomass with species richness, density and basal area.

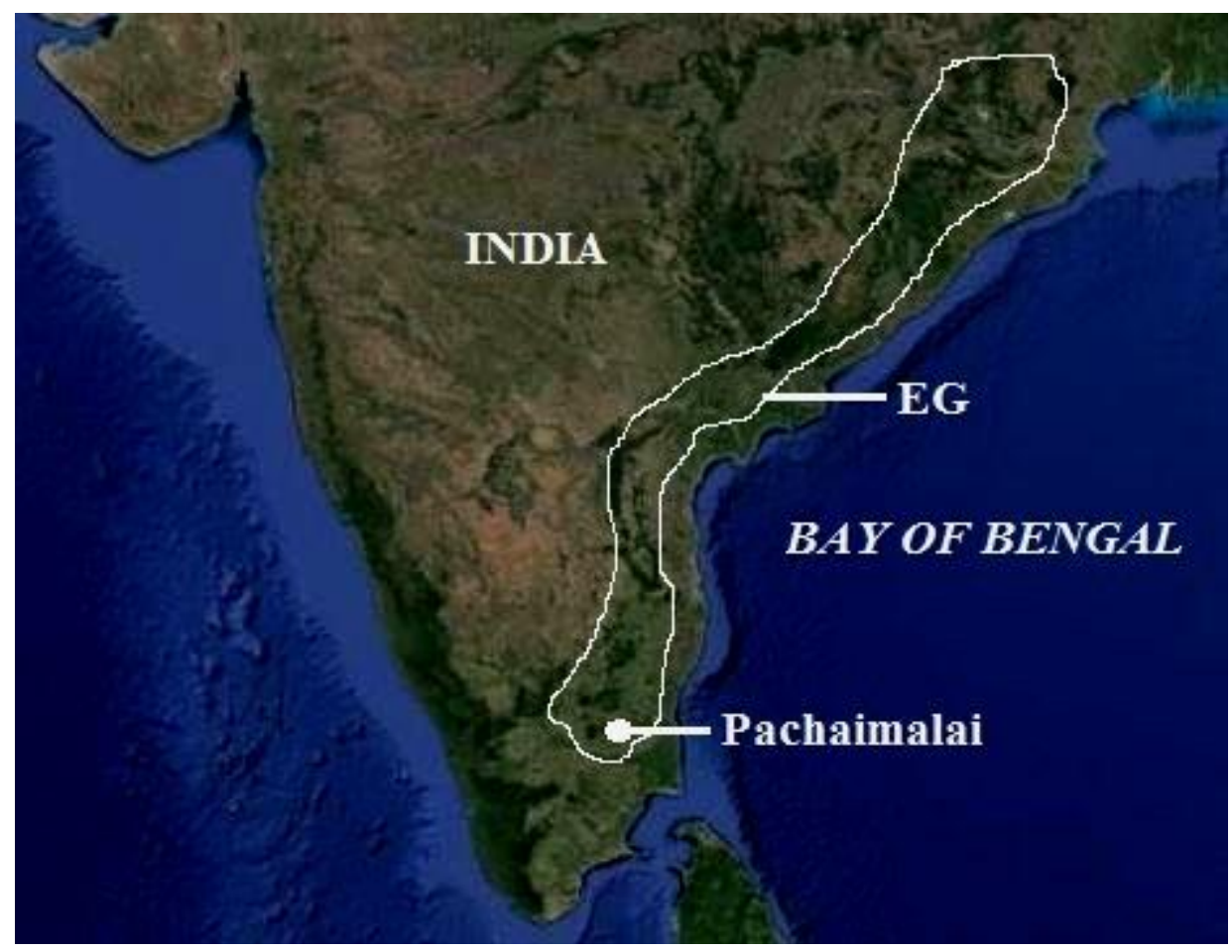

Figure 1. Location of the study area, Pachaimalai forest of the Eastern Ghats in India

\section{Results}

\section{Vegetation structure}

A sum of 5388 trees representing 131 species in 43 families were recorded in twentyfour 0.5 ha transects sampled from the Pachaimalai forest in India. The average species richness per transect was $22 \pm 1.7$ species $( \pm$ S.E.), and it ranged from 12 to 38 species per transect. The tree density per transect was $225 \pm 9.5$ trees $/ 0.5$ ha. The density among the 24 transects was varied from a low of 130 trees $/ 0.5$ ha to a high of 348 trees/ 0.5 ha. The average standing basal area of trees per transect was $6.3 \pm 1.0 \mathrm{~m}^{2} / 0.5$ ha, and it varied from 1.6 to $20.0 \mathrm{~m}^{2} / 0.5$ ha among the 24 transects in Pachaimalai forest.

\section{Forest biomass}

The total aboveground biomass estimated for the 12 ha (24 0.5 ha transects) sampled in Pachaimalai forest was 608.2 tonnes. The average biomass of trees per transect was $25.3 \pm 5.6$ t/0.5 ha, and it ranged from 4.2 to $103.5 \mathrm{t} / 0.5$ ha. Among the 131 species recorded, Nothopegia heyneana (Anacardiaceae) shared a maximum of 116.39 tonnes (19.1\%) to total biomass followed by Canthium dicoccum var. dicoccum (Rubiaceae) (5.9\%), Albizia amara (Mimosaceae) (5.7\%), Gyrocarpus asiaticus (Hernandiaceae) (4.2\%), and Schleichera oleosa (Sapindaceae) (3.8\%) (Table 1). The top five species 
together shared $38.7 \%$ of total aboveground biomass estimated for the 12 ha sampled in Pachaimalai.

Table 1. The aboveground biomass (AGB, in tonnes) of 131 tree species recorded from the twenty four transects (covering 12 ha area) sampled from the Pachaimalai forest

\begin{tabular}{|c|c|c|}
\hline Species (Family) & AGB & $(\%)$ \\
\hline Nothopegia heyneana (Hook.f.) Gamble (Anacardiaceae) & 116.39 & 19.1 \\
\hline $\begin{array}{l}\text { Canthium dicoccum (Gaertn.) Teijsm. and Binn. var. dicoccum } \\
\text { (Rubiaceae) }\end{array}$ & 35.95 & 5.9 \\
\hline Albizia amara (Roxb.) Boivin (Mimosaceae) & 34.92 & 5.7 \\
\hline Gyrocarpus asiaticus Willd. (Hernandiaceae) & 25.31 & 4.2 \\
\hline Schleichera oleosa (Lour.) Oken (Sapindaceae) & 23.01 & 3.8 \\
\hline Vitex altissima L.f. (Verbenaceae) & 22.32 & 3.7 \\
\hline Euphorbia antiquorum L. (Euphorbiaceae) & 20.77 & 3.4 \\
\hline Drypetes roxburghii (Wall.) Hurusawa (Euphorbiaceae) & 20.77 & 3.4 \\
\hline Commiphora caudata (Wight and Arn.) Engler (Burseraceae) & 16.46 & 2.7 \\
\hline Chukrasia tabularis A.Juss. (Meliaceae) & 16.44 & 2.7 \\
\hline Memecylon edule Roxb. (Melastomataceae) & 16.15 & 2.7 \\
\hline Scolopia crenata (Wight and Arn.) Clos (Flacourtiaceae) & 15.60 & 2.6 \\
\hline Diospyros ebenum Koen. (Ebenaceae) & 15.39 & 2.5 \\
\hline Mangifera indica L. (Anacardiaceae) & 14.26 & 2.3 \\
\hline Acacia leucophloea (Roxb.) Willd. (Mimosaceae) & 12.67 & 2.1 \\
\hline Commiphora berryi (Arn.) Engler (Burseraceae) & 9.56 & 1.6 \\
\hline $\begin{array}{l}\text { Anogeissus latifolia (Roxb. ex DC.) Wall. ex Guill. and Perr. } \\
\text { (Combretaceae) }\end{array}$ & 9.52 & 1.6 \\
\hline Acacia planifrons Wight and Arn. (Mimosaceae) & 9.11 & 1.5 \\
\hline Celtis philippensis Blanco (Ulmaceae) & 8.13 & 1.3 \\
\hline Ficus nervosa Heyne ex Roth (Moraceae) & 8.12 & 1.3 \\
\hline Pterospermum xylocarpum (Gaertn.) Sant. and Wagh (Sterculiaceae) & 7.53 & 1.2 \\
\hline Terminalia paniculata Roth (Combretaceae) & 7.43 & 1.2 \\
\hline Chloroxylon swietenia DC. (Flindersiaceae) & 7.37 & 1.2 \\
\hline $\begin{array}{l}\text { Pleiospermium alatum (Wall. ex Wight and Arn.) Swingle } \\
\text { (Rutaceae) }\end{array}$ & 6.10 & 1.0 \\
\hline Syzygium cumini (L.) Skeels (Myrtaceae) & 5.47 & 0.9 \\
\hline Terminalia bellirica (Gaertn.) Roxb. (Combretaceae) & 5.31 & 0.9 \\
\hline Givotia rottleriformis Griff. (Euphorbiaceae) & 5.19 & 0.9 \\
\hline Lannea coromandelica (Houtt.) Merr. (Anacardiaceae) & 4.91 & 0.8 \\
\hline Drypetes sepiaria (Wight and Arn.) Pax and Hoffm. (Euphorbiaceae) & 4.55 & 0.7 \\
\hline Margaritaria indica (Dalz.) Airy Shaw (Euphorbiaceae) & 4.38 & 0.7 \\
\hline Bridelia crenulata Roxb. (Euphorbiaceae) & 4.37 & 0.7 \\
\hline Dolichandrone arcuata (Wight) Clarke (Bignoniaceae) & 3.98 & 0.7 \\
\hline Ficus mollis Vahl (Moraceae) & 3.71 & 0.6 \\
\hline
\end{tabular}


Atalantia monophylla (L.) Correa (Rutaceae)

Pongamia pinnata (L.) Pierre (Papilionaceae)

$3.64 \quad 0.6$

Phyllanthus polyphyllus Willd. (Euphorbiaceae)

$3.58 \quad 0.6$

Acacia farnesiana (L.) Willd. (Mimosaceae)

$3.45 \quad 0.6$

Diospyros ferrea (Willd.) Bakh. var. buxifolia (Rottb.) Bakh.

(Ebenaceae)

$3.39 \quad 0.6$

Strychnos nux-vomica L. (Loganiaceae)

$3.25 \quad 0.5$

Wrightia tinctoria (Roxb.) R.Br. (Apocynaceae)

$3.03 \quad 0.5$

Azadirachta indica A.Juss. (Meliaceae)

$2.97 \quad 0.5$

Mimusops elengi L. (Sapotaceae)

$2.77 \quad 0.5$

Clausena dentata (Willd.) M.Roem. (Rutaceae)

$2.50 \quad 0.4$

Cinnamomum malabatrum (Burm.f.) Blume (Lauraceae)

$2.26 \quad 0.4$

Euodia lunu-ankenda (Gaertn.) Merr. (Rutaceae)

$2.21 \quad 0.4$

Terminalia chebula Retz. (Combretaceae)

$2.15 \quad 0.4$

Cassine glauca (Rottb.) Kuntze (Celastraceae)

$2.10 \quad 0.3$

Manilkara hexandra (Roxb.) Dubard (Sapotaceae)

$2.10 \quad 0.3$

Acacia horrida (L.f.) Willd. (Mimosaceae)

$2.08 \quad 0.3$

Acacia chundra (Roxb. ex Rottl.) Willd. (Mimosaceae)

$2.07 \quad 0.3$

Lepisanthes tetraphylla (Vahl.) Radlk. (Sapindaceae)

$2.03 \quad 0.3$

Aglaia jainii M.V.Viswan. and K.Ramach. (Meliaceae)

$1.98 \quad 0.3$

Ficus drupacea Thunb. var. pubescens (Roth) Corner (Moraceae)

$1.90 \quad 0.3$

Strychnos potatorum L.f. (Loganiaceae)

$1.75 \quad 0.3$

Holoptelea integrifolia (Roxb.) Planch. (Ulmaceae)

$1.71 \quad 0.3$

Diospyros ovalifolia Wight (Ebenaceae)

$1.70 \quad 0.3$

Nothopegia racemosa (Dalz.) Ramam. (Anacardiaceae)

$1.61 \quad 0.3$

Hardwickia binata Roxb. (Caesalpiniaceae

$1.34 \quad 0.2$

Albizia odoratissima (L.f.) Benth. (Mimosaceae)

$1.23 \quad 0.2$

Premna tomentosa Roxb. (Verbenaceae)

$1.22 \quad 0.2$

Pterocarpus marsupium Roxb. (Papilionaceae)

$1.15 \quad 0.2$

Moringa concanensis Nimmo ex Gibs. (Moringaceae)

$1.14 \quad 0.2$

Ziziphus mauritiana Lam. (Rhamnaceae)

$1.01 \quad 0.2$

Diospyros montana Roxb. (Ebenaceae)

$0.97 \quad 0.2$

Chionanthus zeylanica L. (Oleaceae)

$0.93 \quad 0.2$

Canthium dicoccum (Gaertn.) Teijsm. and Binn. var. umbellata

(Wight) Sant. and Merch. (Rubiaceae)

$0.91 \quad 0.2$

Ixora pavetta Andr. (Rubiaceae)

$0.87 \quad 0.1$

Beilschmiedia bourdilloni Brandis (Lauraceae)

$0.82 \quad 0.1$

Citrus medica L. (Rutaceae)

$0.77 \quad 0.1$

Litsea deccanensis Gamble (Lauraceae)

$0.74 \quad 0.1$

Dalbergia latifolia Roxb. (Papilionaceae)

$0.72 \quad 0.1$

Erythroxylum monogynum Roxb. (Erythroxylaceae)

$0.70 \quad 0.1$

Casearia rubescens Dalz. (Flacourtiaceae)

$0.68 \quad 0.1$ 
Tarenna asiatica (L.) Kuntze (Rubiaceae)

Ficus beddomei King (Moraceae)

$0.64 \quad 0.1$

Grewia tenax (Forssk.) Fiori (Tiliaceae)

$0.60 \quad 0.1$

Catunaregam spinosa (Thumb.) Tiruvengadum (Rubiaceae)

$0.60 \quad 0.1$

Alstonia scholaris (L.) R.Br. (Apocynaceae)

$0.59 \quad 0.1$

Erythrina stricta Roxb. (Papilionaceae)

$0.55 \quad 0.1$

Careya arborea Roxb. (Lecythidaceae)

$0.49 \quad 0.1$

Terminalia crenulata Roth (Combretaceae)

$0.49 \quad 0.1$

Bauhinia racemosa Lam. (Caesalpiniaceae)

$0.48 \quad 0.1$

Sapindus emarginatus Vahl. (Sapindaceae)

$0.48 \quad 0.1$

Alseodaphne semicarpifolia Nees var. semecarpifolia (Lauraceae)

$0.47 \quad 0.1$

Antiaris toxicaria (Pers.) Lesch. (Moraceae)

$0.45 \quad 0.1$

Atalantia racemosa Wight and Arn. (Rutaceae)

$0.44 \quad 0.1$

Limonia acidissima L. (Rutaceae)

$0.41 \quad 0.1$

Filicium decipiens (Wight and Arn.) Thw. (Sapindaceae)

$0.39 \quad 0.1$

Spondias pinnata (L.f.) Kurz (Anacardiaceae)

$0.38 \quad 0.1$

Mallotus philippensis (Lam.) Muell.-Arg. (Euphorbiaceae)

$0.36 \quad 0.1$

Dolichandrone atrovirens (Heyne ex Roth) Spangue (Bignoniaceae)

$0.35 \quad 0.1$

Mallotus stenanthus Muell.Arg. (Euphorbiaceae)

$0.33 \quad 0.1$

Dichrostachys cinerea (L.) Wight and Arn. (Mimosaceae)

Bischofia javanica Blume (Bischofiaceae)

$0.23<0.1$

Ficus microcarpa L.f. (Moraceae)

Madhuca longifolia (Koen.) Macbr. var. longifolia (Sapotaceae)

$0.21<0.1$

$0.21<0.1$

$0.20<0.1$

Ehretia pubescens Benth. (Cordiaceae)

$0.17<0.1$

Streblus asper Lour. (Moraceae)

Morinda pubescens J.E. Smith (Rubiaceae)

$0.16<0.1$

Albizia lebbeck (L.) Benth. (Mimosaceae)

$0.15<0.1$

$0.11<0.1$

Cipadessa baccifera Miq. (Meliaceae)

Benkara malabarica (Lam.) Tirvengadum (Rubiaceae)

$0.10<0.1$

Cassia fistula L. (Caesalpiniaceae)

$0.09<0.1$

Murraya koenigii (L.) Spreng. (Rutaceae)

$0.09<0.1$

$0.08<0.1$

Diospyros barberi Ramaswami (Ebenaceae)

$0.08<0.1$

Flacourtia indica (Burm.f.) Merr. (Flacourtiaceae)

$0.07<0.1$

Ochna obtusata DC. (Ochnaceae)

$0.07<0.1$

Rhus mysorensis G. Don (Anacardiaceae)

Alangium salviifolium (L.f.) Wang. (Alangiaceae)

$0.07<0.1$

Streblus taxoides (Heyne ex Roth) Kurz (Moraceae)

$0.07<0.1$

Capparis grandis L. (Capparaceae)

$0.06<0.1$

Polyalthia cerasoides (Roxb.) Bedd. Nakulsi (Annonaceae)

$0.05<0.1$

Ziziphus xylopyrus (Retz.) Willd. (Rhamnaceae)

$0.04<0.1$

Santalum album L. (Santalaceae)

$0.04<0.1$

$0.04<0.1$

Maytenus emarginata (Willd.) Ding Hou (Celastraceae)

$0.04<0.1$ 


\begin{tabular}{lrc} 
Ficus benghalensis L. (Moraceae) & 0.04 & $<0.1$ \\
Symplocos cochinchinensis (Lour.) Moore (Symplocaceae) & 0.04 & $<0.1$ \\
Diospyros melanoxylon Roxb. (Ebenaceae) & 0.04 & $<0.1$ \\
Sterculia foetida L. (Sterculiaceae) & 0.03 & $<0.1$ \\
Buchanania axillaris (Desr.) Ramam. (Anacardiaceae) & 0.03 & $<0.1$ \\
Cleistanthus collinus Benth. ex Hook.f. (Euphorbiaceae) & 0.03 & $<0.1$ \\
Euphorbia nivulia Buch.-Ham. (Euphorbiaceae) & 0.03 & $<0.1$ \\
Gardenia resinifera Roth (Rubiaceae) & 0.03 & $<0.1$ \\
Firmiana colorata (Roxb.) R.Br. (Sterculiaceae) & 0.02 & $<0.1$ \\
Solanum erianthum D.Don (Solanaceae) & 0.02 & $<0.1$ \\
Murraya paniculata (L.) Jack (Rutaceae) & 0.02 & $<0.1$ \\
Chionanthus mala-elengi (Dennst.) P.S.Green (Oleaceae) & 0.02 & $<0.1$ \\
Rapanea wightiana (Wall. ex DC.) Mez (Myrsinaceae) & 0.02 & $<0.1$ \\
Diospyros humilis Bourd. (Ebenaceae) & 0.02 & $<0.1$ \\
Euonymus indicus Heyne ex Roxb. (Celastraceae) & 0.02 & $<0.1$ \\
Antidesma zeylanicum Lam. (Euphorbiaceae) & 0.02 & $<0.1$ \\
Total & 608.24 & 100 \\
\hline
\end{tabular}

\section{Size class distribution}

All the trees sampled in Pachaimalai forest were categorized into eight size classes viz. $30-60 \mathrm{~cm}$ class, $60-90 \mathrm{~cm}, 90-120 \mathrm{~cm}, 120-150 \mathrm{~cm}, 150-180 \mathrm{~cm}, 180-210 \mathrm{~cm}, 210-$ $240 \mathrm{~cm}$ and $>240 \mathrm{~cm}$ class, based on their girth measurement at breast height. Contribution of aboveground biomass was maximum $(31.1 \%)$ for $60-90 \mathrm{~cm}$ class followed by $30-60 \mathrm{~cm}(22.2 \%), 90-120 \mathrm{~cm}$ and $120-150 \mathrm{~cm}$ (Fig. 2).

The stand basal area was dominated by $30-60 \mathrm{~cm}$ size class $(35.9 \%)$, followed by $60-$ $90 \mathrm{~cm}(33.8 \%), 90-120 \mathrm{~cm}$ and $120-150 \mathrm{~cm}$ class (Fig. 3). In case of density, the smallest class $30-60 \mathrm{~cm}$ alone contributed more than seventy six per cent of the total trees recorded from 12 ha area sampled from the Pachaimalai forest (Fig. 4).

\section{Regression analysis}

The relationships of aboveground biomass with species richness, density and basal area of trees were analyzed through linear regression (Fig. 5). The analysis showed that tree biomass were positively correlated with species richness $\left(R^{2}=0.702\right)$ and basal area $\left(R^{2}=0.979\right)$, for the 24 transects sampled in Pachaimalai forest (Fig. 5). Whereas, no significant relationship was predicted between biomass and density of trees $\left(R^{2}=\right.$ 0.320) in the Pachaimalai forest of Eastern Ghats (Fig. 5). 


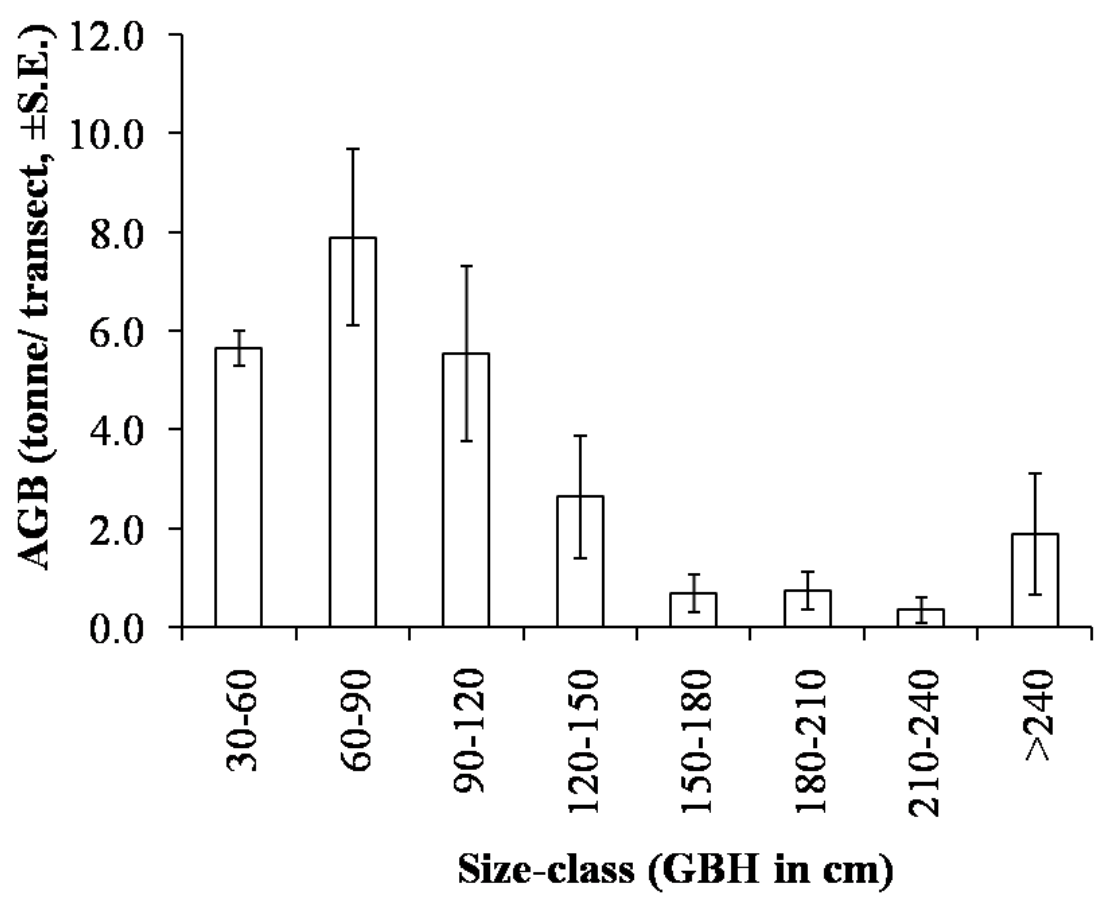

Figure 2. The average aboveground biomass (AGB, in tonnes) of trees categorized into eight size classes for the twenty four 0.5 ha transects sampled at the Pachaimalai forest

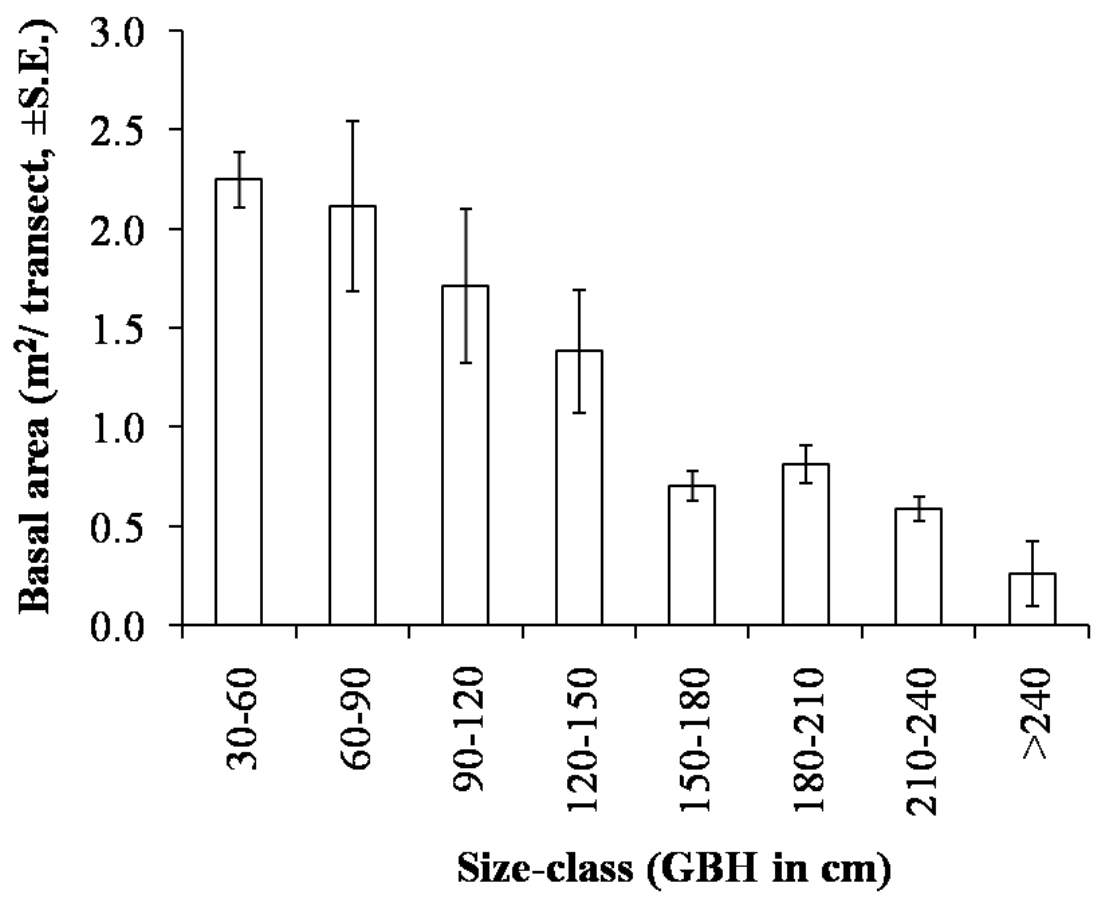

Figure 3. The average basal area of trees categorized into eight size classes for the twenty four 0.5 ha transects sampled at the Pachaimalai forest 


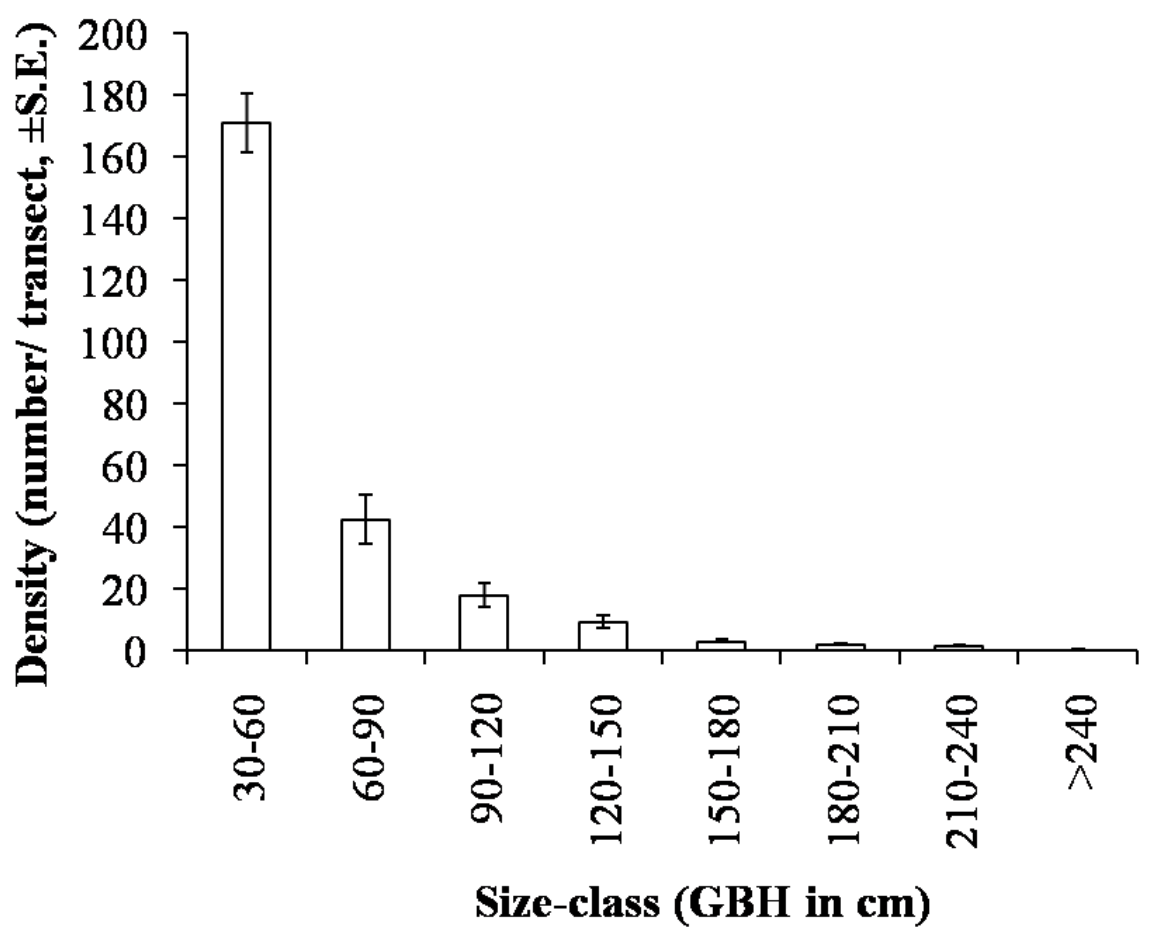

Figure 4. The average density of trees categorized into eight size classes for the twenty four 0.5 ha transects sampled at the Pachaimalai forest

\section{Discussion}

During the last decade, climate change has increased the need of quantification on the amount of forest biomass in different regions of the globe for climate policy definition (Djomo et al., 2010). The results of this study reveal that the aboveground biomass of the Pachaimalai forest of the Eastern Ghats in India (50.7 t/ha) was moderate and falls within the range reported for other tropical forests of the world (Table 2). It was almost five folds greater than the biomass value reported for rainforest at Bajo Calima, Choco, Columbia (Fabre-Langendoen and Gentry, 1991), but ten folds lesser than the value reported for the rain forests in New Guinea (Edwards and Grubb, 1977).

The order of aboveground biomass contribution of trees categorized into eight size classes in Pachaimalai forest were $60-90 \mathrm{~cm}>30-60 \mathrm{~cm}>90-120 \mathrm{~cm}>120-150 \mathrm{~cm}>$ above $240 \mathrm{~cm}>180-210 \mathrm{~cm}>150-180 \mathrm{~cm}>210-240 \mathrm{~cm}$ class. The smaller size classes $(30-60 \mathrm{~cm}$ to $90-120 \mathrm{~cm}$ class) contributed seventy five percent of the total aboveground biomass. This reveals that the standing biomass stock of the Pachaimalai forest was dominated by the young trees.

The regression analysis revealed a strong positive relationship that the biomass of trees increased with an increase in basal area and species richness, but not with density (Fig. 5). This shows that basal area and species richness of forest stands were good indicators for the stand biomass rather than stand density of Pachaimalai forest, and a similar trend was reported in Murali et al. (2005). 


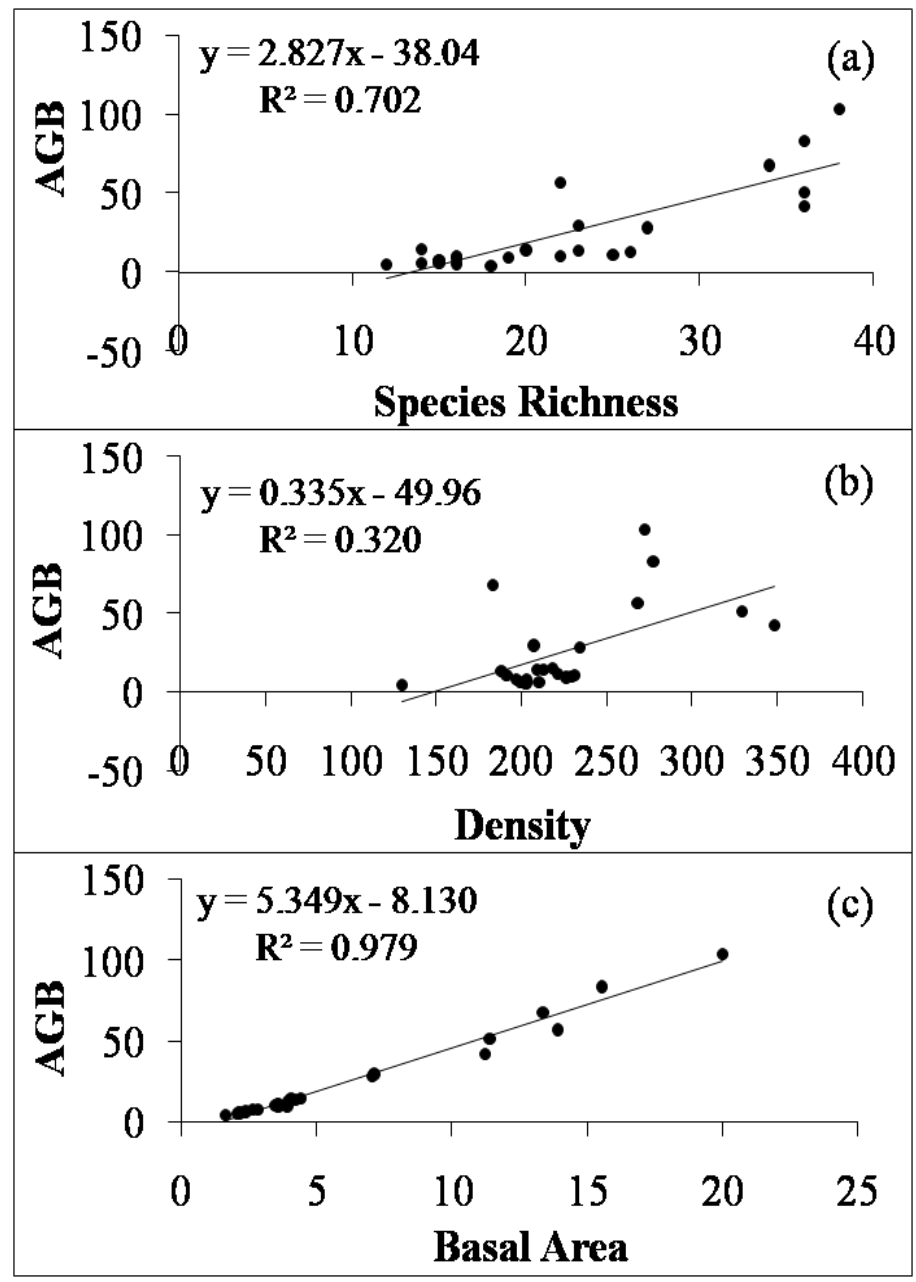

Figure 5. The relationships between aboveground biomass (AGB) and species richness (a), density $(b)$ and basal area (c) for the twenty four transects sampled at Pachaimalai forest

Table 2. Comparison of aboveground biomass (AGB, t/ha) of Pachaimalai forest with other forests of the world.

\begin{tabular}{lllll}
\hline Forest type and location & AGB & BA & DN & RS \\
\hline Tropical forests, Pachaimalai forest, India & 57.7 & 12.5 & 449 & PS \\
Rainforest, Bajo Calima, Choco, Columbia & 193.29 & 25.01 & 664 & A \\
Rainforest, Bajo Calima, Choco, Columbia & 168.71 & 22.48 & 704 & A \\
Rainforest, Bajo Calima, Choco, Columbia & 17.63 & 5.4 & 2820 & A \\
Rainforest, Bajo Calima, Choco, Columbia & 11.09 & 4.9 & 2000 & A \\
Tropical dry evergreen forest, Rayapatti, India & 73.06 & 12.4 & 889 & B \\
Tropical moist forests, Bangladesh & $85-206$ & - & - & C \\
Tropical dry evergreen forest, Arasadikuppam, & 90.25 & 17.6 & 2813 & B \\
India & & & & \\
Tropical dry evergreen forest, & 90.25 & 16.9 & 1349 & B \\
Kuzhanthaikuppam, India & & & & \\
Tropical dry evergreen forest, Maramadakki, & 91.06 & 15.5 & 724 & B
\end{tabular}


India

Tropical dry evergreen forest, Araiyapatti, India $103.68 \quad 19.1 \quad 807 \quad$ B

Tropical dry evergreen forest, Oorani, India

Tropical dry evergreen forest,

$\begin{array}{llll}113.46 & 27.3 & 1284 & \text { B }\end{array}$

Shanmuganathapuram, India

Tropical dry evergreen forest, Thirumanikkuzhi,

127.91

$22.1 \quad 1663 \quad$ B

India

Tropical dry evergreen forest, Karisakkadu, India

Tropical moist forests, Bangladesh

$\begin{array}{llll}134.13 & 29.3 & 1077 & B\end{array}$

Tropical dry evergreen forest, Puthupet, India

Montane rain forests, Jamaica

Tropical rain forests, Malaysia

Lower Montane forests, El Verde, Puerto Rico

$138.73 \quad 21.6 \quad 596 \quad$ B

$150-210 \quad-\quad-\quad D$

$\begin{array}{llll}173.1 & 36.5 & 1567 & \text { B }\end{array}$

229-312 - - E

230-290 - -

Tropical seasonal rain forest, Xishuangbanna,

China

Tropical rain forests, Khade, Ghana

$233.4 \quad-\quad-\quad G$

Tropical rain forests, New Guinea

$\begin{array}{llll}241.27 & 31.28 & 730 & \mathrm{H}\end{array}$

Tropical rain forests, Khado Chang, Thailand

$\begin{array}{lllll}265.8 & - & - & \mathrm{I}\end{array}$

$286 \quad-\quad-\quad$ J

Tropical rain forest, Cambodia

$290.6 \quad-\quad-\quad$ K

Tropical rain forests, Western Ghats, India

348-415 - $\quad-\quad$ L

Montane rain forests, New Guinea

$458.2 \quad-\quad-\quad-M$

505

BA-Basal area ( $\mathrm{m}^{2} / \mathrm{ha}$ ), DN-density (stems/ha); RS-References; PS-Present study; A-Fabre-Langendoen and Gentry, 1991; B-Mani and Parthasarathy, 2007; C- Drigo et al., 1988; D- Milde et al., 1985; E-Tanner, 1975; F-Whitmore, 1975; G-Jordon, 1981; H-Shanmughavel et al., 2001; I-Greenland and Gowel, 1970; J-Enright, 1979; K-Kira et al., 1974; L-Hozumi et al., 1979; M-Rai, 1984; N-Edwards and Grubb, 1977

Estimation of forest biomass is an essential aspect of studies for quantification of forest carbon stocks and their effects on the global carbon balance (Ketterings et al., 2001). Hence, the present study provides valuable base line biomass data helpful for quantification of carbon stocks of Pachaimalai forest of Eastern Ghats which in turn would help for modeling forest carbon stocks at national as well as global level.

Acknowledgements. I sincerely thank Dr. N. Parthasarathy, Professor of Ecology and Environmental Sciences, Pondicherry University, Puducherry, for all his support and encouragements.

\section{REFERENCES}

[1] Alamgir, M., Al-Amim, M. (2008): Allometric models to estimate biomass organic carbon stock in forest vegetation. -Journal of Forest Research 19: 101-106.

[2] Brown, S., Schroeder, P.E. (1999): Spatial patterns of aboveground production and mortality of woody biomass for eastern US forests. -Ecological Applications 9: 968-980.

[3] Brown, S., Gillespie, A.J.R., Lugo, A.E. (1989): Biomass estimation methods for tropical forests with applications to forest inventory data. -Forest Science 35: 881-902.

[4] Chavan, B.L., Rasal, G.B. (2010): Sequestered standing carbon stock in selective tree species grown in University campus at Aurangabad, Maharashtra, India. -International Journal of Engineering Science and Technology 2: 3003-3007.

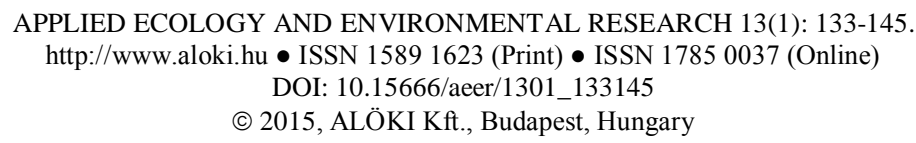


[5] Chave, J., Condit, R., Lao, S., Caspersen, J.P., Foster, R.B., Hubbell, S.P. (2003): Spatial and temporal variation in biomass of a tropical forest: results from a large census plot in Panama. -Journal of Ecology 91: 240-252.

[6] Chave, J., Andalo, C., Brown, S., Cairns, M.A., Chambers, J.Q., Eamus, D., Folster, H., Fromard, F., Higuchi, N., Kira, T., Lescure, J.-P., Nelson, B.W., Ogawa, H., Puig, H., Riera, B., Yamakura, T. 2005. Tree allometry and improved estimation of carbon stocks and balance in tropical forests. -Oecologia 145: 87-99.

[7] Clark, D.B., Clark, D.A. (2000): Landscape-scale variation in forest structure and biomass in a tropical rain forest. -Forest Ecology and Management 137: 185-198.

[8] Crow, T.R. (1978): Common regressions to estimate tree biomass in tropical stands. Forest Science 24: 110-114.

[9] Djomo, A.N., Ibrahima, A., Saborowski, J., Gravenhorst, G. (2010): Allometric equations for biomass estimations in Cameroon and pan moist tropical equations including biomass data from Africa. -Forest Ecology and Management 260: 1873-1885.

[10] Drigo, R., Shaheduzzaman, Md., Chowdhury, J.A. (1988): Inventory of forest resources of south Sylhet forest division. Assistance to the forestry section - phase ii. FAO/UNDP project BGD/85/085, field document no. 3, Rome, Italy.

[11] Edwards, P.J., Grubb, P.J. (1977): Studies of mineral cycling in a Montane Rain forest in New Guinea, I-Distribution of organic matter in the vegetation and soil. -Journal of Ecology 65: 1943-1969.

[12] Enright, N.J. (1979): Litter production and nutrient partitioning in rain forests near Bulolo, Papua New Guinea. -Malayan Forester 42: 202-220.

[13] Fabre-Langendoen, D., Gentry, A.H. (1991): The structure and diversity of rain forests at Bajo Calima, Choco region, western Columbia. -Biotropica 23: 2-11.

[14] Greenland, D.J., Gowel, J.M.L. (1970): Nutrient content of the moist tropical forests of Guana. -Plant Science 12: 154-174.

[15] Houghton, R.A., Lawrence, K.L., Hackler, J.L., Brown, S. (2001): The spatial distribution of forest biomass in the Brazilian Amazon: a comparison of estimates. -Global Change Biology 7:731-746.

[16] Hozumi, K., Yoda, K., Kira, T. (1979): Production ecology of tropical rain forests in south western Cambodia. II. Photosynthetic function in an evergreen seasonal forest. Nature and Life in Southeast Asia 6: 57-81.

[17] Jordan, C.F. (1981): Productivity of tropical forest and its relation to a energy storage. Journal of Ecology 59: 127-142.

[18] Ketterings, Q.M., Coe, R., van Noordwijk, M., Ambagau, Y., Palm, C.A. (2001): Reducing uncertainty in the use of allometric biomass equations for predicting aboveground tree biomass in mixed secondary forests. -Forest Ecology and Management 146: 199-209.

[19] Kira, T., Ogawa, H., Yoda, K., Ogina, K. (1974): Primary production by a tropical rain forest of southern Thailand. -Botanical Magazine 77: 425-429.

[20] Laurance, W.F., Laurance, S.G., Ferreira, L.V., Merona, J.M.R., Gascon, C., Lovejoy, T.E. (1997): Biomass collapse in Amazonian forest fragments. Science 278: 1117-1118.

[21] Mani, S., Parthasarathy, N. (2007): Above-ground biomass estimation in ten tropical dry evergreen forest sites of peninsular India. -Biomass and Bioenergy 31: 284-290.

[22] Milde, de.R., Shaheduzzaman, Md., Drigo, R. (1985): The high forest in Chittagong district. Vols 1 and 2, FAO/UNDP Project BGD/79/017, field document no.3, Rome, Italy.

[23] Mitra, A., Sengupta, K., Banerjee, K. (2011): Standing biomass and carbon storage of above-ground structures in dominant mangrove trees in the Sundarbans. -Forest Ecology and Management 261: 1325-1335.

[24] Murali, K.S., Bhat, D.M., Ravindranath, N.H. (2005): Biomass estimation equations for tropical deciduous and evergreen forests. -International Journal of Agricultural Resources, Governance and Ecology 4: 81-92.

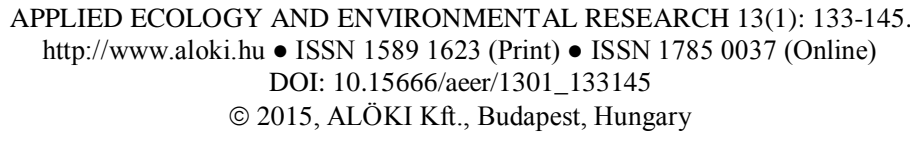


[25] Murphy, P.G., Lugo, A.E. (1986): Structure and biomass of a subtropical dry forest in Puerto Rico. -Biotropica 18: 89-96.

[26] Nascimento, H.E.M., Laurance, W.F. (2002): Total aboveground biomass in central Amazonian rainforests: a landscape-scale study. -Forest Ecology and Management 168: 311-321.

[27] Nath, A.J., Das, G., Das, A.K. (2009): Above ground standing biomass and carbon storage in village bamboos in North East India. -Biomass and Bioenergy 33: 1188-1196.

[28] Negi, J.D.S., Chauhan, P.S., Negi, M. (2003): Evidences of climate change and its impact on structure and function of forest ecosystems in and around doon vally. -Indian Forester 129: 757-769.

[29] Pragasan, L.A., Parthasarathy, N. (2010): Landscape-level tree diversity assessment in tropical forests of southern Eastern Ghats, India. -Flora 205: 728-737.

[30] Rai, S.N. (1984): Above ground biomass in tropical rain forests of Western Ghats, India. Indian Forester 8: 754-764.

[31] Salazar, S., Sanchez, L.E., Galindo, P., Santa-Regina, I. (2010): Above-ground tree biomass equations and nutrientpools for a paraclimax chestnut stand and for a climaxoak stand in the Sierra de Francia Mountains, Salamanca, Spain. -Scientific Research and Essays 5: 1294-1301.

[32] Satto, T., Madgwick, H.A.I. (1982): Forest Biomass. Martinus Nishoff, Boston, M.A., USA.

[33] Segura, M. (2005): Allometric models for tree volume and total aboveground biomass in tropical humic forest in Costa Rica. Biotropica, 37: 2-8.

[34] Shanmughavel, P., Zheng, Z., Liqiing, S., Min, C. (2001): Floristic structure and biomass distribution of a tropical seasonal rain forest in Xishuangbanna, southwest China. Biomass and Bioenergy 21: 165-175.

[35] Shepashenko, D., Shvidenko, A., Nilsson, S. (1998): Phytomass (live biomass) and carbon of Siberian forests. -Biomass and Bioenergy 14:21-31.

[36] Tanner, E.V.J. (1975): Studies on the biomass productivity in a series of montane rain forests in Jamaica. -Journal of Ecology 68: 578-588.

[37] Tiwari, A.K., Singh, J.S. (1987): Analysis of forest landuse and vegetation in a part of central Himalaya, using aerial photographs. -Environment Conservation Journal 14: 233244.

[38] Verwijst, T., Telenius, B. (1999): Biomass estimation procedures in short rotation forestry. -Forest Ecology and Management 121: 137-146.

[39] Whitmore, T.C. (1975): Tropical rain forests of the far east. - Clarendon Press, Oxford 